\title{
Update on Etripamil Nasal Spray for the At-home Treatment of Acute Paroxysmal Supraventricular Tachycardia
}

\author{
Gavin S Chu ${ }^{1}$ and Dhiraj Gupta ${ }^{1,2}$
}

1. Liverpool Heart and Chest Hospital NHS Foundation Trust, Liverpool, UK; 2. Faculty of Health and Life Sciences, University of Liverpool, Liverpool, UK

DOI: https://doi.org/10.17925/HI.2021.15.1.2

$\mathrm{T}$ he current treatment of sustained paroxysmal supraventricular tachycardia (PSVT) often requires attendance at a medical facility. This burden is driven by the lack of an effective self-administered treatment for PSVT. Etripamil (Milestone Pharmaceuticals, Saint-Laurent, QC, Canada) is a novel intra-nasal preparation of a rapidly effective but short-acting calcium-channel blocker, which shows promise in offering out-of-hospital treatment for patients with PSVT. Studies, to date, have demonstrated good tolerability and potential efficacy, with a safety profile that is acceptable for unsupervised self-administration. This article reviews the current epidemiology and international guidelines for the treatment of acute PSVT, the pharmacology and clinical trial evidence behind the novel agent etripamil, and considers its potential role in the management of patients with PSVT.

\section{Keywords}

Paroxysmal supraventricular

tachycardia, etripamil nasal spray

Disclosure: Gavin S Chu and Dhiraj Gupta have no financial or non-financial relationships or activities to declare in relation to this article.

Review Process: Double-blind peer review.

Compliance with ethics: This study involves a review of the literature and did not involve any studies with human or animal subjects performed by any of the authors.

Authorship: The named authors meet the International Committee of Medical Journal Editors (ICMJE) criteria

for authorship of this manuscript, take responsibility

for the integrity of the work as a whole, and have

given final approval for the version to be published.

Access: This article is freely accessible at

touchCARDIO.com (C) Touch Medical Media 2021

Received: 21 January 2021

Accepted: 17 March 2021

Published online: 15 July 2021

Citation: Heart International. 2021;15(1):2-6

Corresponding author: Dhiraj Gupta,

Liverpool Heart and Chest Hospital,

Thomas Drive, Liverpool, L14 3PE, UK.

E: Dhiraj.Gupta@|hch.nhs.uk

Support: No funding was received in

the publication of this article.
Paroxysmal supraventricular tachycardia (PSVT) defines a group of cardiac arrhythmias where the underlying mechanism is dependent upon structures at or above the level of the atrioventricular node. The recent PRE-EMPT study retrospectively evaluated health records of over 4.2 million people in the USA, estimating PSVT incidence at 73/100,000, and national prevalence at 168/100,000 (translating into some 393,810 affected American adults), with older and female individuals being more at risk. ${ }^{1}$ On the other side of the Atlantic, the UK Biobank prospective population cohort study, looking at the records of 502,627 people aged 40-69, showed a baseline prevalence of PSVT at $0.28 \%$, overall prevalence of $0.42 \%$, and an incidence of 0.51 cases per 1,000 person-years. ${ }^{2}$

The National Hospital Ambulatory Medical Care Survey estimated that in the USA, 50,000 emergency department visits per year were attributed to PSVT, at a cost upward of US\$190 million. ${ }^{3}$ More recently, a retrospective analysis of claims data for a PSVT cohort against a propensity-matched non-PSVT group confirmed higher rates of emergency department attendance and a substantial post-diagnosis increase in healthcare expenditure that was not attributable to ablation therapy in patients with PSVT. ${ }^{4}$ This article will first provide an update on the current international guideline recommendations for the management of haemodynamically stable PSVT, followed by a review of the clinical evidence and potential utility of etripamil nasal spray (Milestone Pharmaceuticals, Saint-Laurent, Montreal, QC, Canada), a novel agent which shows promise for the acute treatment of PSVT.

\section{The current management of paroxysmal supraventricular tachycardia}

The typical patient with PSVT presents to acute medical services with sudden onset symptoms, such as palpitations, dizziness and or breathlessness, and electrocardiography (ECG) demonstrating a narrow complex tachycardia. Current international guidelines for the acute management of haemodynamically stable narrow complex tachycardia recommend first-line therapy with manoeuvres causing para-sympathetic nervous system stimulation (Figure 1).5.6 These can terminate PSVT, but their effectiveness ranges from 19\% to 54\% in randomized controlled trials.? Besides the limited efficacy, the manoeuvres themselves are not entirely free of risk, with neurological complications, including cerebral infarction, reported in up to $0.45 \%$ in some series. ${ }^{8-10}$ second-line recommended treatment using adenosine has an effectiveness of around $90 \%$ and has the advantage of both rapidity of effect and a very short half-life, but requires intravenous administration and, therefore, hospital attendance.11 Third-line recommended treatment with verapamil can be as effective as adenosine, but carries the risks of excess negative chronotropism and negative inotropism, and also requires intravenous administration..$^{12,13}$ 
Figure 1: Summary of current international recommendations for the management of haemodynamically stable acute regular narrow complex tachycardia ${ }^{5,6}$

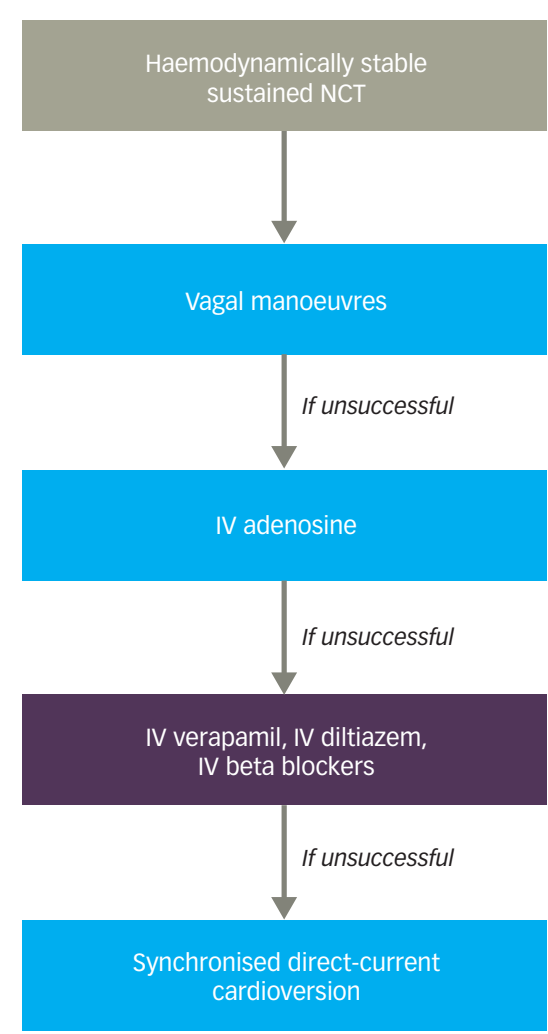

Blue boxes indicate class I recommendations. Purple box indicates class lla recommendations.

IV = intravenous; $N C T$ = narrow complex tachycardia

Data sourced from Page et al. $2016^{5}$ and Brugada et al. 2020.

The use of oral medications is not part of current guideline recommendations for acute PSVT episodes. This is reflective of their slow onset of action (in one study, the mean time to arrhythmia conversion was 32 minutes for a combination of diltiazem and propranolol, 74 minutes for flecainide, and 77 minutes for placebo), as well as the recognized risk of proarrhythmia with antiarrhythmic drugs. ${ }^{14,15}$

Beyond the acute setting, catheter ablation is a class I recommended treatment for PSVT, with success rates $>95 \%$ and recurrence rates $<5 \%$. 5,6 Although largely safe, catheter ablation carries a low but finite risk of major complications, including $<1 \%$ risk of permanent atrioventricular block. Those not proceeding to ablation default to a watch-and-wait approach, with or without concurrent oral pharmacotherapy. In summary, current pharmacological therapies for acute PSVT mandate hospital attendance and therefore increase the burden of care for both patients and their healthcare systems.

\section{Etripamil: A novel potential outpatient treatment for paroxysmal supraventricular tachycardia Pharmacology}

Etripamil is a novel intra-nasal verapamil analogue, which shows promise for the rapid outpatient treatment of PSVT. It is a short-acting non-dihydropyridine phenylalkylamine class L-type calcium channel antagonist, with a time-to-peak plasma concentration of 8 minutes, and a half-life of 20 minutes. The drug is metabolized by ubiquitous serum esterases, and its major metabolite is an inactive carboxylic acid.
Etripamil slows atrioventricular nodal conduction and prolongs atrioventricular nodal refractory periods via the inhibition of slow inward calcium channels. High-density electroanatomic mapping has further suggested that etripamil causes loss of voltage in the slow pathway bridge with gradual recovery, mirroring observed changes in atrioventricular block cycle length. ${ }^{16}$

The potent combination of a convenient intra-nasal mode of delivery and rapid onset of action, as well as a short half-life, make etripamil an attractive proposition for patient self-administration outside of the formal healthcare environment. The use of etripamil is directed towards the two most common subtypes of PSVT that involve the atrioventricular node: atrioventricular nodal re-entrant tachycardia (AVNRT) and atrioventricular re-entrant tachycardia (AVRT), which together account for up to $90 \%$ of PSVT cases. ${ }^{17}$

\section{Phase I evidence}

Two phase I studies have been completed. The first-in-human phase I study evaluated a single intra-nasal dose of etripamil in two formulations. Pharmacokinetic analysis demonstrated rapid absorption and elimination, and dose-dependent PR prolongation was observed, consistent with its proposed AV nodal effects..$^{18}$ All adverse events were mild, no serious adverse events were reported, and in particular, no prolongation of the QT interval was observed. ${ }^{19}$

In the second (NODE-102), there were no significant differences in etripamil plasma levels or pharmacodynamic outcomes between Caucasian volunteers or subjects of Japanese descent. Secondary analyses across all patients showed the relevant pharmacodynamic effects with $70 \mathrm{mg}$ etripamil for PSVT, as measured by PR interval prolongation on ECG, was approximately 5-50 minutes..$^{20}$ Dose-dependent PR prolongation was consistent with its proposed atrioventricular nodal effects, without any effect on QRS duration or QT interval. ${ }^{21}$

\section{Phase II evidence}

The phase II, randomized, placebo-controlled, double-blind, dose-ranging NODE-1 study (Efficacy and Safety of Intranasal MSP-2017 [Etripamil] for the Conversion of PSVT to Sinus Rhythm; ClinicalTrials. gov identifier: NCT02296190) involved 104 patients undergoing invasive cardiac electrophysiology testing across 34 North American centres. ${ }^{21,22}$ The median age was 55 years (range 19-85). PSVT (87\% AVNRT) was induced in all 104 patients. After a sustained arrhythmia of at least 5 minutes, the study drug was given intra-nasally, with an endpoint of cardioversion within 15 minutes of drug administration. Doses used were placebo, $35 \mathrm{mg}, 70 \mathrm{mg}, 105 \mathrm{mg}$ and $140 \mathrm{mg}$, with 35\%, 65\%, 87\%, 75\% and $95 \%$ reaching the defined endpoint, respectively. A statistically significant difference in the rate of conversion to sinus rhythm was detected for the top three doses of etripamil when compared against placebo, with evidence of plateauing beyond $70 \mathrm{mg}$ according to a maximal efficacy dose-response model $\left(R^{2}=0.915\right)$. The median time to cardioversion in each of the top three dosing groups was $<3$ minutes.

No major adverse events were seen that led to study termination. The most common adverse events were often attributable to local irritation, including oropharyngeal pain, cough, rhinorrhoea, nasal congestion, dysgeusia and lacrimation. One patient (from the $35 \mathrm{mg}$ group) experienced facial flushing, dyspnoea and chest discomfort; one patient (in the $105 \mathrm{mg}$ group) had nausea and vomiting; and one (in the $105 \mathrm{mg}$ group) experienced severe cough.

One patient in the $140 \mathrm{mg}$ dosing group cardioverted immediately to sinus rhythm, but then developed second degree atrioventricular block with 
hypotension after a further 5 minutes, which resolved after 43 minutes. Patients who received $70 \mathrm{mg}$ or less of etripamil did not experience any significant hypotensive effect.

\section{Phase III evidence}

On the basis of the above, the $70 \mathrm{mg}$ dose of etripamil has been taken forward to three phase III clinical trials. NODE-301 (ClinicalTrials.gov Identifier: NCT03464019) was a randomized, double-blind, placebo-controlled, efficacy and safety study in 77 centres across the USA and Canada. ${ }^{23}$ Patients with ECG-documented PSVT sustained for at least 20 minutes were randomized to either etripamil or placebo. The test drug was self-administered upon onset of suspected PSVT outside of hospital which did not terminate with a vagal manoeuvre, and was coupled with up to 5 hours of ambulatory ECG monitoring to provide independent adjudication of suspected PSVT events. The primary endpoint was pre-defined as termination of an episode of PSVT to sinus rhythm for at least 30 seconds within the 5-hour period following study drug administration. The study was event-driven, with a pre-specified analysis planned once more than 150 positively-adjudicated PSVT events treated with etripamil/placebo reached the primary endpoint.

The early results of NODE-301 have been reported. ${ }^{20,24}$ Out of 431 recruited subjects, 10 (2.3\%) failed an initial medically supervised etripamil test dose (this and other important exclusion criteria are elaborated in Table 1). ${ }^{23}$ Four hundred and nineteen patients were finally randomized in a $2: 1$ fashion to etripamil or placebo. The trial failed to demonstrate superiority of etripamil over placebo in terminating PSVT over the pre-specified 5-hour period following study drug administration. However, as the half-life of etripamil is substantially less than 5 hours, a post-hoc analysis was performed looking at only the first 45 minutes following use of the study drug, and showed higher rates of PSVT termination with etripamil (61\%), as opposed to placebo (45\%). Two cases of first-degree atrioventricular block occurred in the etripamil group, with otherwise no difference in the safety profile between etripamil and placebo.

An extension of NODE-301, the RAPID study (Efficacy and Safety of Etripamil for the Termination of Spontaneous PSVT; EudraCT 2018-000308-41; ClinicalTrials.gov Identifier: NCT03464019) is currently underway, which will comprise an ongoing safety evaluation, and also incorporate an arm with two doses, administered 10-15 minutes apart. ${ }^{23}$ This better mirrors PSVT treatment practice in the acute setting (e.g., the use of consecutive escalating bolus doses of adenosine), and is expected to benefit a broader group of patients..$^{20,25}$

NODE-302 (Safety Study of Intranasal Etripamil for the Termination of Spontaneous Episodes of Paroxysmal Supraventricular Tachycardia (PSVT); ClinicalTrials.gov Identifier: NCT03635996) is a multi-centre open-label extension study of NODE-301, which has finished recruitment and will further report on the safety of additional doses of etripamil. ${ }^{26}$ NODE-303 (Safety Study of Etripamil Nasal Spray for Patients With Paroxysmal Supraventricular Tachycardia; ClinicalTrials.gov Identifier: NCT04072835)is an ongoing international study to evaluate the safety and efficacy of using $70 \mathrm{mg}$ doses of etripamil after failure of vagal manoeuvres, outside of the clinical setting, on multiple SVT episodes. ${ }^{27}$ Centres across the Americas, South Africa and Europe are involved.

Table 2 summarizes the clinical studies of intra-nasal etripamil that

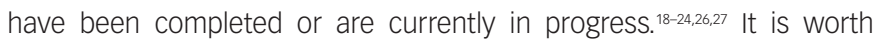
noting that patients on beta blockers or calcium channel antagonists have been included in the phase III clinical trials, which should broaden the generalizability of their findings by encompassing patients who are already using currently recommended first-line pharmacological therapy.
Table 1: Key exclusion criteria for the NODE-301 phase III clinical trial of etripamil23

\begin{tabular}{l} 
Adverse effect from a medically supervised test dose of etripamil: \\
- symptoms of severe hypotension \\
- $\mathrm{BP}$ drop $\geq 40 \mathrm{mmHg}$ \\
- $\mathrm{BP}<75 \mathrm{mmHg}$ \\
- sinus bradycardia $\leq 40 \mathrm{bpm}$ \\
- sinus pause $\geq 3$ seconds \\
- second- (Mobitz II) or third-degree AV block \\
- ventricular arrhythmias/>6 ventricular ectopic beats over 45 seconds \\
- atrial fibrillation $>30$ seconds \\
\hline History of non-AV nodal dependent tachycardia (e.g., atrial fibrillation) \\
\hline Ventricular pre-excitation \\
\hline Second- or third-degree AV block \\
\hline Systolic BP < 90 mmHg at screening \\
\hline History of severe hypotension or syncope with PSVT \\
\hline Stroke within the 6 months prior to randomization \\
\hline Heart failure NYHA class II-IV \\
\hline Amiodarone use within 30 days of randomization \\
\hline Digoxin, class I or class III anti-arrhythmic drug use <5 half-lives prior to \\
randomization
\end{tabular}

$A V=$ atrioventricular $; B P=$ blood pressure; $b p m=$ beats per minute; $N Y H A=$ New York Heart Association; PSVT = paroxysmal supraventricular tachycardia.

\section{Anticipated clinical utility of etripamil nasal spray for paroxysmal supraventricular tachycardia}

\section{Alternative to catheter ablation}

For some patients, the perceived advantages of catheter ablation are outweighed by the perceived risks. This may apply particularly to those whose PSVT symptoms are very infrequent. At present, such patients who decline catheter ablation have limited choice beyond that of vagal manoeuvres and ongoing oral medication either regularly or on a "pill-in-the-pocket" basis. For this group, as-needed intra-nasal etripamil may represent an appealing alternative approach to rapidly terminating their symptoms.

\section{Bridge to catheter ablation}

In some healthcare systems, there may be a substantial delay between referral for catheter ablation and the procedure itself. Etripamil could serve as an appropriate short-term alternative or possibly even adjunct to oral medications, acting as a 'bridge to ablation' in this context.

\section{To facilitate reduction or cessation of oral medications}

Some patients with PSVT may not wish to take regular oral medications. Even if initiated, it is not uncommon for them to inquire if such regular medications could be discontinued, whether to due side effects or for other reasons. Etripamil may facilitate this.

\section{Emergency department use}

With the positive safety profile and rapidity of conversion demonstrated by etripamil, it could be utilized acutely within emergency departments following ECG confirmation of PSVT, circumventing (or reducing the urgency of) intravenous access for definitive treatment. ${ }^{21,22}$ In this situation, treatment success would not only be therapeutic, but also indicate to the patient the utility of intra-nasal etripamil for future PSVT episodes; therefore, potentially reducing hospital visits. 
Table 2: Summary of human clinical studies using intra-nasal etripamil for paroxysmal supraventricular tachycardia that are complete or are currently in progress $18-24,26,27$

\begin{tabular}{|c|c|c|}
\hline & Main outcomes measured & Status \\
\hline \multicolumn{3}{|l|}{ Phase I } \\
\hline First-in-human ${ }^{18,19}$ & $\begin{array}{l}\text { Confirmed rapid absorption and elimination of IN etripamil. Dose-dependent effect on PR } \\
\text { prolongation }\end{array}$ & Complete \\
\hline NODE-102 20 & $\begin{array}{l}\text { No difference in pharmacodynamic outcomes between Caucasians and subjects of Japanese } \\
\text { descent. Dose-dependent effect on PR prolongation }\end{array}$ & Complete \\
\hline \multicolumn{3}{|l|}{ Phase ॥ } \\
\hline $\begin{array}{l}\text { NODE-1 } \\
(\text { NCT02296190) })^{21,22}\end{array}$ & $\begin{array}{l}\text { Significant difference in arrhythmia conversion rates compared to placebo, dose-effect plateau at } \\
70 \mathrm{mg}\end{array}$ & Complete \\
\hline \multicolumn{3}{|l|}{ Phase III } \\
\hline $\begin{array}{l}\text { NODE-301 Part } 1 \\
(\text { NCT03464019) } \\
\text { (20,23,24 }\end{array}$ & $\begin{array}{l}\text { Superior conversion rates compared to placebo in post-hoc analysis at } 45 \text { minutes, but not for the } \\
\text { pre-specified endpoint at } 5 \text { hours }\end{array}$ & Complete \\
\hline $\begin{array}{l}\text { NODE-301 Part } 2 \text { (RAPID; } \\
\text { NCT03464019; EudraCT } \\
\text { 2018-000308-41)20,22,23 }\end{array}$ & Time to arrhythmia conversion; efficacy of a repeat dosing protocol & Expected end January 2022 \\
\hline $\begin{array}{l}\text { NODE-302 } \\
(\text { NCT03635996) }\end{array}$ & $\begin{array}{l}\text { Safety and efficacy evaluation of self-administration for multiple PSVT episodes (extension of } \\
\text { NODE-301) }\end{array}$ & Complete \\
\hline $\begin{array}{l}\text { NODE-303 } \\
(\text { NCT04072835) }\end{array}$ & Safety and efficacy evaluation of self-administration outside of the clinical setting & Expected end August 2021 \\
\hline
\end{tabular}

IN = intra-nasal; PSVT = paroxysmal supraventricular tachycardia

\section{Failure or unsuitability of catheter ablation}

Catheter ablation for AVNRT and AVRT has a very high success rate (>95\%), but there are circumstances where it may not be successful or may be associated with a prohibitively high risk. ${ }^{28}$ The latter includes patients with para-Hisian accessory pathways, for example, where the close proximity of the accessory pathway to the atrioventricular node precludes safe ablation. ${ }^{29}$ Intra-nasal etripamil may be useful in these niche circumstances.

\section{Future directions}

The first patient for the RAPID study was enrolled towards the end of 2020, and top-line data are expected in early 2022. In combination with NODE-301, these data may fulfil efficacy requirements for approval by the United States Food and Drug Administration..$^{25}$ NODE302 has completed recruitment, with results anticipated in the near future. At the time of writing, NODE-303 is estimated to complete at the end of August 2021. However, recruitment to NODE-303 is being affected by the current COVID-19 pandemic, with some centres closing to enrollment as a result. It is anticipated that this, together with increased focus on the RAPID study, will lead to downsizing of the NODE-303 trial. ${ }^{20}$

Intra-nasal etripamil shows promise as a treatment for PSVT, but may remain a niche product in view of the fact that the first-choice therapy for most patients is (usually curative) catheter ablation. Further investigation will be required to establish whether or not etripamil could also be effective for other conditions which respond to calcium channel blockade, such as angina or atrial fibrillation.

\section{Conclusion}

The lack of an effective self-administered product for PSVT increases the burden on acute medical services. Etripamil is a novel intra-nasal preparation of a rapidly effective, but short-acting, calcium channel blocker that shows promise in offering out-of-hospital treatment for patients with PSVT. Studies, to date, have demonstrated good tolerability and potential efficacy, with a safety profile that is acceptable for unsupervised self-administration. The results from ongoing clinical trials are eagerly awaited. $\square$
1. Go AS, Hlatky MA, Liu TI, et al. Contemporary burden and correlates of symptomatic paroxysmal supraventricular tachycardia. J Am Heart ASSOC. 2018;7:e008759.

2. Khurshid S, Choi SH, Weng L, et al. Frequency of cardiac rhythm abnormalities in a half million adults. Circ Arrhythmia Electrophysiol. 2018;11:e006273

3. Murman DH, MCDonald AJ, Pelletier AJ, Camargo CA. U.S. emergency department visits for supraventricular tachycardia, 1993-2003. Acad Emerg Med. 2007:14:578-81.

4. Sacks NC, Cyr PL, Preib MT, et al. Healthcare resource use and expenditures in patients newly diagnosed with paroxysmal supraventricular tachycardia. Am I Cardiol. 2020;125:215-21.

5. Page RL, Joglar JA, Caldwell MA, et al. 2015 ACC/AHA/ HRS Guideline for the management of adult patients with supraventricular tachycardia: Executive Summary: A report of the American College of Cardiology/American Heart Association Task Force on Clinical Practice Guidelines and the Heart Rhythm Society. Circulation. 2016;133:e471-505.

6. Brugada J, Katritsis DG, Arbelo E, et al. 2019 ESC Guidelines for the management of patients with supraventricular tachycardia. Eur Heart J. 2020;41:655-720.

7. Smith GD, Fry MM, Taylor D, et al. Effectiveness of the Valsalva manoeuvre for reversion of supraventricular tachycardia. Cochrane Database Syst Rev. 2015:2015:CD009502.
8. Mariaca AF, Valdueza JM, Gaebel C, Gomez-Choco M. Simultaneous transient global amnesia and right MCA stroke after Valsalva manoeuvre. BMJ Case Rep. 2017;2017:bcr2016218990

9. Ungvari Z, Yabluchanskiy A, Tarantini S, et al. Repeated Valsalva maneuvers promote symptomatic manifestations of cerebral microhemorrhages: implications for the pathogenesis of vascular cognitive impairment in older adults. Geroscience. vascular cognitive im

10. Walsh $T$, Clinch D, Costelloe $A$, et al. Carotid sinus massage How safe is it? Age Ageing. 2006:35:518-20.

11. DiMarco JP, Sellers TD, Berne RM, et al. Adenosine: Electrophysiologic effects and therapeutic use for terminating paroxysmal supraventricular tachycardia. Circulation. 1983;68:1254-63

12. Delaney B, Loy J, Kelly A. The relative efficacy of adenosine versus verapamil for the treatment of stable paroxysmal supraventricular tachycardia in adults. Eur J Emerg Med. 2011;18:148-52.

13. Alabed S, Sabouni A, Providencia R, et al. Adenosine versus intravenous calcium channel antagonists for supraventricular tachycardia Cochrane Database Syst Rev. 2017:10:CD005154.

14. Alboni P, Tomasi C, Menozzi C, et al. Efficacy and safety of out-of-hospital self-administered single-dose oral drug treatment in the management of infrequent, well-tolerated paroxysmal supraventricular tachycardia. J Am Coll Cardio. 2001;37:548-53

15. Lafuente-Lafuente $C$, Valembois $L$, Bergmann J-F, Belmin J. Antiarrhythmics for maintaining sinus rhythm after cardioversion of atrial fibrillation. Cochrane Database Syst Rev. 2015;CD005049

16. Choe WC, Sundaram S, Boorman C, et al. High-density mapping of the slow pathway in a patient with atrioventricular nodal reentry given intranasal etripamil during the NODE-1 study. reentry given intranasal etripamil during the

17. Orejarena $L A$, Vidaillet $H$, DeStefano $F$, et al. Paroxysmal supraventricular tachycardia in the general population. J Am Coll Cardiol. 1998;31:150-7.

18. United States Securities and Exchange Commission Form S-1 - Registration Statement under the Securities Act of 1933. 2019. Available at: www.sec.gov/Archives/edgar/data/000 1408443/000104746919002213/a2238338zs-1.htm (accessed 14 April 2021).

19. Milestone Pharmaceuticals. Milestone announces positive phase 1 data for MSP-2017; supports advancement into phase 2 in PSVT patients. 2014. Available at: https://investors milestonepharma.com/news-releases/news-release-details/ milestone-announces-positive-phase-1-data-msp-2017- 
supports (accessed 20 April 2021)

20. United States Securities and Exchange Commission. Form 10-Q - Quarterly Report Pursuant to Section 13 or 15(d) of the Securities Exchange Act of 1934, for the Quarterly Period Ended September 30, 2020. 2020. Available at: www.sec.gov/ Archives/edgar/data/1408443/000155837020013774/mistArchives/edgar/data/1408443/00015583702

21. Stambler BS, Dorian P, Sager PT, et al. Etripamil nasal spray for rapid conversion of supraventricular tachycardia to sinus rhythm. J Am Coll Cardiol. 2018;72:489-97.

22. ClinicalTrials.gov. Efficacy and safety of intranasal MSP-2017 (Etripamil) for the conversion of PSVT to sinus rhythm (NODE-1). ClinicalTrials.gov Identifier: NCT02296190. Available at: https://clinicaltrials.gov/ct2/show/NCT02296190 (accessed 9 July 2021).

23. ClinicalTrials.gov. Efficacy and safety of etripamil for the termination of spontaneous PSVT. NODE 301 [Part 1 and Part 2 (The RAPID Study)]. ClinicalTrials.gov Identifier: NCT03464019. (trialsgov/ct2/show/NCT03464019 (accessed 20 May 2021).

24. Stambler BS Plat F Sager PT, et al. Etripamil nasal spray for acute termination of spontaneous episodes of paroxysmal supraventricular tachycardia (NODE-301). Heart Rhythm. 2020;17:1200. Abstract D-LBCT01-01.

25. Milestone Pharmaceuticals. Milestone Pharmaceuticals announces first patient enrolled in RAPID, a pivotal phase 3 study of etripamil in PSVT. 2020. Available at: https://investors, milestonepharma.com/news-releases/news-release-details/ milestone-pharmaceuticals-announces-first-patient-enrolledrapid (accessed 20 April 2021)

26. ClinicalTrials.gov. Safety study of intranasal etripamil for the termination of spontaneous episodes of paroxysmal supraventricular tachycardia (PSVT). NODE-302. ClinicalTrials. gov Identifier: NCT03635996. Available at: https://clinicaltrials. gov/ct2/show/NCT03635996 (accessed 20 May 2021).

27. ClinicalTrials.gov. Safety study of etripamil nasal spray for patients with paroxysmal supraventricular tachycardia. NODEpatients with paroxysmal supraventricular tachycardia. NODE303. Clinicalitrials.gov Identifier. NCTO4072835. Avallable at: https://clinicaltrials.gov/ct2/show/NCT04072835 (accessed 20 May 2021)

28. Brachmann J, Lewalter T, Kuck KH, et al. Long-term symptom improvement and patient satisfaction following catheter ablation of supraventricular tachycardia: insights from the German ablation registry. Eur Heart J. 2017;38:1317-26.

29. Brugada J, Puigfel M, Mont L, et al. Radiofrequency ablation of anteroseptal, para-Hisian, and mid-septal accessory pathways using a simplified femoral approach. Pacing Clin Electrophysiol. 1998;21:735-41. 